\title{
OPTIMIZING THE WOODPULP STOWAGE USING LAGRANGEAN RELAXATION WITH CLUSTERS
}

\author{
Glaydston Mattos Ribeiro ${ }^{1}$ \\ DCCI - Department of Computer Science \\ UniAracruz - Faculty of Aracruz \\ R. Prof. Berilo Basílio dos Santos, 180 - Vila Rica. Zip Code 29194-910 \\ Aracruz - ES - Brazil \\ Tel: +55 27 3256-1102 FAX: +55 27 3256-1102 \\ E-mail: glaydston@fsjb.edu.br \\ Luiz Antonio Nogueira Lorena ${ }^{2}$ \\ LAC - Computer and Applied Mathematics Laboratory \\ INPE - Brazilian Space Research Institute \\ Av. dos Astronautas,1.758 - Jd. Granja. ZIP Code: 12.227-010 \\ São José dos Campos - SP - Brazil \\ Tel: +55 12 3945-6555 FAX: +55 12 3945-6357 \\ E-mail: $\left\{\right.$ glaydston $^{1}$, lorena $\left.^{2}\right\}$ @lac.inpe.br
}

\begin{abstract}
The cargo stowage process in ships consists in arranging items into holds. This paper approaches the problem of finding the maximum number of stowed units of woodpulp into holds of dedicated maritime international ships. This problem, essentially three-dimensional can be reduced for the two-dimensional case due to constraints provided by the transport, and becomes similar to the manufacturer's pallet loading problem. We present in this paper a formulation to the woodpulp stowage solved by a lagrangean relaxation with clusters (LagClus) that considers the conflict graph generated by overlaps of woodpulp units. Computational tests are performed and compared with the real results obtained in Brazilian ports. The results obtained by LagClus were better than the real results, and consequently, it can provide savings if we look at the shipping logistics costs.
\end{abstract}

Keywords: Sea Transport, Logistics, Cost Benefit. 


\section{INTRODUCTION}

Cargo stowage consists in arranging items (units of cargo) into a transportation unit in view of the most economic conveyance (Branch, 1996). The stowage planning must consider the kind of cargo to be loaded, the kind of transportation unit, nature of cargo, among other factors (Handabaka, 1994).

We consider in this work the woodpulp stowage problem in holds of dedicated maritime international ships. Figure 1 (a) presents a sketch of a woodpulp unit or simply unit, (b) presents the holds through the ship, and in (c) is presented a possible stowage of one hold.

The Woodpulp Stowage Problem (WSP), essentially, can be defined as a three-dimensional packing problem of units into a large box (hold). A woodpulp unit is composed of a set of small bales, where each one is composed of cellulose sheets that are tied with a wire. In the literature, the WSP can be seen as a particular case of the Three-Dimensional Bin Packing Problem (3D-BPP). In 3D-BPP we have a set of $n$ items, each one has a width $\left(w_{i}\right)$, height $\left(h_{i}\right)$ and length $\left(l_{i}\right)$ for all $i \in I=\{1,2, \ldots, n\}$, and a number of limited bins (boxes), which initially we consider that there are at least $n$ three-dimensional identical bins with the same dimensions, $\mathrm{i}$. e., width $(W)$, height $(H)$ and length $(L)$. The 3D-BPP aims to obtain an orthogonal packing of all items $n$ using the smallest number of bins as possible.

In WSP, all units to be stowed present the same dimensions, and they are positioned in one hold at a time. Thus, this problem can be reduced to the case of packing identical items in one hold. Besides, the stowage is achieved by tiers of units, using cranes. The number of tiers is limited by the hold's height.

Figure 1: Woodpulp unit (a), layout of the holds (b), and (c) a stowage solution.

Each woodpulp unit is also tied by a wire (See Figure 1 (a)). It allows an efficient shipment with the cranes at commencement. However, during the transportation with the balance yielded by ship, the units can move changing positions between two tiers. If the new positions produced crossed wires they can be ruptured and the cargo will be damaged, or even worse 
they can produce fire. Thus, to avoid these crossed wires and consequently damages, in practical operations once the first tier is defined, all remaining tiers are identical to this first one, and the WSP can be reduced to the two-dimensional bin packing problem.

According to the typology proposed by Dyckhoff (1990), the WSP can be classified as 2/B/O/C (two-dimensional, selection of items, one object, identical items) therefore it can be seen as a manufacturer's pallet loading problem (MPLP) (Hodgson, 1982), a well-known problem in the literature. The MPLP consists in arranging the maximum number of identical boxes into a pallet. Considering their practical applications (Morabito et al, 2000), several optimization methods have been developed for MPLP. The exact algorithms work, basically, with a tree search structure (Dowsland, 1987; Bhattacharya et al, 1998; Alvarez-Valdez et al, 2005b). Heuristics can be constructive, dividing the pallet in blocks (Young-Gun and MaingKyu, 2001; Smith and De Cani, 1980), recursive methods (Morabito and Morales, 1998) and techniques based in identified structures known as G4 (Scheithauer and Terno, 1996) and L (Lins et al, 2003; Birgin et al, 2005). Some other works applied metaheuristics, such as Tabu Search (Pureza and Morabito, 2006; Alvarez-Valdes et al, 2005a) and Genetic Algorithms (Herbert and Dowsland 1996). There is also lagrangean relaxation as can be seen in Morabito and Farago (2002) and in Ribeiro and Lorena (2005a).

However, if we look at the MPLP and WSP from another point of view, they are equivalent to the classic Maximum Independent Set Problem (MISP) (Dowsland, 1987). Ribeiro and Lorena (2005a) explored the relationship of MPLP and MISP to propose a new lagrangean relaxation approach to MPLP. A conflict graph is first generated considering overlapping of items and is subsequently partitioned in clusters. The lagrangean relaxation with clusters (LagClus) considers the relaxing of the constraints (edges) in different clusters producing quality bounds.

The WSP can be represented by a conflict graph where each vertex corresponds to the leftlower-corner of the one woodpulp unit in the hold, and the edges indicate the possible overlaps between woodpulp units. The conflict graph can be partitioned (Ribeiro and Lorena, 2005b), and this work follows the approach of Lorena and Ribeiro (2005a), presenting a formulation for the WSP and applying a LagClus. The LagClus was tested on real instances 
for the WSP obtained at some Brazilian ports. The results found for the WSP were better than practical solutions used at these ports, allowing 70 more woodpulp units per hold to be transported.

The structure of the paper is as follows. The next section presents a formulation for WSP based on the two-dimensional non-guillotine cutting problem proposed by Beasley (1985) and the LagClus steps to solve the WSP. After that, we present the computational results for the real instances. Finally, some comments are given.

\section{FORMULATION OF THE WSP}

Let $L$ and $W$ be the hold length and width, respectively, such that $L \geq W$, and, $l$ and $w$, the woodpulp unit length and width, respectively, such that $l \geq w$ and $l \leq \operatorname{Min}(L, W)$. To represent all possible ways of packing a woodpulp unit, let $\left(l_{1}, w_{1}\right)=(l, w)$ and $\left(l_{2}, w_{2}\right)=(w, l)$. Thus, these possible positions can be represented by $\left(l_{i}, w_{i}\right)_{i=1,2}$ that indicates the unit length and width considering the orientation $i$. Figure 2 presents these possible orientations onto the hold's floor.

Figure 2: Possible orientations of one woodpulp unit.

To represent the woodpulp unit positions onto the hold's floor, let $X$ and $Y$ be two sets that are used to define the coordinates $(p, q)$ of the unit left-lower-corner. These sets can be described by:

$$
\begin{aligned}
& X=\left\{p \in Z^{+} \mid p=\sum_{i=1}^{2} l_{i} b_{i}, 0 \leq p \leq L-w, b_{i} \geq 0 \text { and integer, } i=1,2\right\} \\
& Y=\left\{q \in Z^{+} \mid q=\sum_{i=1}^{2} w_{i} b_{i}, 0 \leq q \leq W-w, b_{i} \geq 0 \text { and integer, } i=1,2\right\}
\end{aligned}
$$

These sets were introduced by Christofides and Whitlock (1977) and they are called normal sets. The restriction of the woodpulp unit positions to these sets does not imply loss of generality. 
Let $a$ be a function that describe overlapping constraints between units. This function can be obtained in advance for each vertex $(p, q)$ in relation to some other vertex $(r, s)$, for each orientation $i$, where $p \in X\left|p \leq L-l_{i}, q \in Y\right| q \leq W-w_{i}, r \in X, s \in Y$, and $i=1,2$. Thus, this function can be expressed by:

$$
a_{i p q r s}=\left\{\begin{array}{l}
1, \text { If } 0 \leq p \leq r \leq p+l_{i}-1 \leq L-1 \text { and } 0 \leq q \leq s \leq q+w_{i}-1 \leq W-1 \\
0, \text { Otherwise }
\end{array}\right.
$$

Now, let $x_{i p q} \in\{0,1\}$ be a binary decision variable for all $p \in X\left|p \leq L-l_{i}, q \in Y\right| q \leq W-w_{i}$, and $i=1,2$. If $x_{i p q}=1$, one woodpulp unit is placed in hold coordinates $(p, q)$ with orientation $i$, otherwise, $x_{i p q}=0$.

Then the WSP can be formulated as a particular case of the Beasley (1985) formulation:

$$
v(W S P)=\operatorname{Max}\left(\sum_{i=1}^{2} \sum_{\left\{p \in X \mid p \leq L-l_{i}\right\}} \sum_{\left\{q \in Y \mid q \leq W-w_{i}\right\}} x_{i p q}\right)
$$

Subject to:

$$
\begin{aligned}
& \sum_{i=1}^{2} \sum_{\left\{p \in X \mid p \leq L-l_{i}\right\}} \sum_{\left\{q \in Y \mid q \leq W-w_{i}\right\}} a_{i p q r s} x_{i p q} \leq 1, \quad \forall r \in X \text { and } s \in Y \\
& x_{i p q} \in\{0,1\} \forall i=1 \ldots 2, p \in X \text { such that } p \leq L-l_{i} \text {, and } q \in Y \text { such that } q \leq W-w_{i}
\end{aligned}
$$

The constraints set (5) avoids overlapping between positions and each constraint is a clique that ensures that a particular "square" is covered by at most one unit. The constraint set (6) ensures that all variables are binaries.

The WSP graph of conflicts is created directly from formulation (4) - (5), where overlappings represent edges and units are the corresponding vertices. The conflict graph presents some grouped areas (clusters) that do not have influence in other areas, as for instance, the hold lower-left-corner has a disposition of units that do not affect the opposite side, the hold upperright-corner. Thus, using this characteristic, the LagClus is created in the following lines: 
a) Create the conflict graph from WSP formulation and apply a graph partitioning heuristic to divide the conflict graph into $\bar{P}$ clusters. This step generates $\bar{P}$ subgraphs (sub-problems);

b) Relax the constraints present in the WSP formulation that correspond to vertices in different clusters. In each relaxed clique, verify if there are pairs of vertices that are in the same cluster, and if they exist, add to each respective cluster one adjacent constraint between each pair found;

c) The lagrangean relaxation obtained is divided into $\bar{P}$ sub-problems and solved.

Figure 3: Example of how decompose a clique to be relaxed.

Note what happens at step b) and consider Figure 3. If some clique constraint is relaxed (constraint defined in (5)), it must be decomposed and each one of its edges must be analyzed. If some edge is connecting two vertices in the same cluster, it must be appended to the respective cluster. This procedure is essential to make the relaxation stronger and to avoid an invalid solution for some cluster. Figure 3 shows exactly this approach. Note that if we do not add $x_{1}+x_{2} \leq 1$ to Cluster 1 and $x_{3}+x_{4} \leq 1$ to Cluster 2, both clusters could provide invalid solutions, i. e., $x_{1}$ and $x_{2}$ could appear together in solution of the Cluster 1 as well as $x_{3}$ and $x_{4}$ in solution of the Cluster 2.

Figure 4: Lagrangean relaxation with clusters. (a) Conflict graph, (b) edges connecting the clusters, and (c) clusters or sub-problems.

The example in Figure 4 explains the entire process of the LagClus. Figure 4(a) has a conflict graph with two well-defined clusters. Figure 4(b) shows all edges connecting the clusters that are relaxed in LagClus, and Figure 4(c) shows the two sub-graphs (or two sub-problems) similar to the original problem that can be separated and solved independently.

For the computational tests, we have implemented a subgradient algorithm to solve the lagrangean dual (Parker and Rardin, 1988; Narciso and Lorena, 1998). At the subgradient optimization process, when all sub-problems are solved, we re-grouped these sub-problems to 
update the lagrangean multipliers, and again all sub-problems are separated and solved independently, and so on until the algorithm reaches some stopping condition.

The step size control in the algorithm was the one proposed by Held and Karp (1971), beginning with 2 and halving it whenever the upper bound does not decrease for 30 successive iterations. The stopping tests used are: step less than or equal to 0.005 ; difference between the best lower and upper bounds less than 1; and the length of the subgradient vector equal to zero. The lagrangean multipliers are initialized with zero.

Before the first iteration of the subgradient algorithm, we used a simpler form of the block heuristic proposed by Smith and De Cani (1980) for the MPLP to generate an initial solution for the WSP. This solution is used in step size definition and can be substituted by a solution provided by the LagClus, made feasible to WSP. This heuristic, called VI, identifies all vertices present in the relaxed solution that are in conflict, removing from this solution the vertex with the largest number of vertices in conflict. This process is repeated until the heuristic produces a feasible solution to WSP. After that, it tries to introduce other vertices in this solution aiming to get the maximum number of independent vertices. These other vertices are the remaining vertices, not including the first vertices removed from the relaxed solution. The VI heuristic is shown in Figure 5. The step sizes of the subgradient algorithm are updated considering the LagClus solutions and the feasible solutions obtained with VI.

Figure 5 - Verify and improvement heuristic used in LagClus process.

\section{COMPUTATIONAL RESULTS}

The computational experiments were conducted on some real instances of WSP, obtained with Brazilian ports. Table 1 shows the instances data, where columns $H$ and $h$ represent the hold height and woodpulp unit height, respectively. The last column indicates the practical solution used at the ports. Observe that the largest instances in the literature have 150 boxes at maximum for the related MPLP problem (Alvarez-Valdez et al, 2005 ${ }^{\mathrm{b}}$ ).

Table 1: Real instances of the WSP obtained at Brazilian ports. 
The formulation shown for the WSP was applied over the real problems reported at Table 1, and the CPLEX 7.5 was used to prove the optimality. However, it was not possible to ensure the optimal solutions after 10 hours of execution. So, we used the LagClus to approximately solve the WSP.

Figure 6 depicts an example of conflict graph representing the instance L2. Note that there are many edges $(4,792,819)$ that complicate our visualization of several areas. This graph allowed us to feel the complexity of the WSP.

Figure 6 - Conflict graph generated for the instance L2: 22,870 vertices and 4,792,819 edges.

Table 2 shows the results provided with the LagClus. The second column indicates the number of clusters used in the partition phase, the "Area Bound" is given by $\left\lfloor\left(L^{*} W\right) /\left(l^{*} w\right)\right\rfloor$ (where $\lfloor z\rfloor$ denotes rounding down to the nearest integer), the "Barnes bound" is provided by the formula described in Barnes (1979), the "Lower bound" is the bound found by the VI heuristic, the "Upper bound" is the bound provided by LagClus, and the column "Gap (\%)" indicates the gap found between the lower and upper bounds:

$$
\text { Gap }=\frac{(\text { Upper Bound }- \text { Lower Bound })}{\text { Upper Bound }} * 100
$$

The number of clusters was obtained previously. We analyzed the tradeoff between the quality of the upper bounds and the computational times. We used a number of clusters that provide good upper bounds with an acceptable time.

Our results for these instances of the WSP were interesting. For the instance L2 we obtained a better upper bound than the area bound. For the instances L3, L4 and L7 the LagClus provided upper bounds better than the area and the Barnes bound together. In only one instance was the upper bound not good, the instance L15. However, note that all these results can be better if we reduce the number of clusters.

Table 2: LagClus computational results for real instances of the WSP. 
Table 3 compares our results with the practical results used by Brazilian ports. The column "Difference" represents the difference between our lower bounds and those practical solutions. The column "Number of tiers" is calculated using $\lfloor H / h\rfloor$.

As can be seen, the average lower bound provided by the LagClus yielded a stowage that has 8.67 more woodpulp units per tiers and 69.33 (1804.44 - 1735.11) more woodpulp units per hold. Considering that a ship has on average 10 holds, we are able to transport more 693.3 woodpulp units per trip. Given that one ship realizes several trips in one year, the transportation amount in one year with our solutions can generate a significant decrease in the logistic shipping costs.

Table 3: Results for the real instances of the Brazilian ports.

Figure 7 shows stowage plans used at the ports and the stowage plans provided by LagClus. The portion gray indicates wasted areas. Note that visually our solutions are better than the solutions used at the ports.

Figure 7 - Solutions provided by LagClus for real instances of the WSP.

The code in $\mathrm{C}++$ and the tests are performed in a computer with Pentium IV processor with $512 \mathrm{MB}$ of RAM memory. The sub-problems, either for LagClus or for sub-problems, were solved by CPLEX 7.5 (ILOG, 2001). For the graph partitioning task, we have used METIS (Karypis and Kumar, 1998) that is a well-known heuristic for graph partitioning problems. Given a conflict graph $G$ and a pre-defined number $\bar{P}$ of clusters, the METIS divides the graph in $\bar{P}$ clusters minimizing the number of edges with terminations in different clusters.

\section{CONCLUSIONS}

This work presented the woodpulp stowage problem (WSP) in holds of dedicated maritime ships. The problem is reduced to a two-dimensional case and consequently a mathematical formulation based on the formulation for the two-dimensional non-guillotine cutting problem 
proposed by Beasley (1985) can be adapted to the WSP. CPLEX 7.5 was not able to solve all real instances with this new formulation. Thus, we developed and used a lagrangean relaxation with clusters (LagClus) to obtain good bounds.

The computational results over real instances of the WSP showed that LagClus heuristic provided better results than the original practical results, in some cases almost one unit of the optimal. The duality gaps were less than $1.44 \%$. When we compared our results in a whole stowage of one hold, our results have shown that we can create stowage plans with approximately 70 units more than the practical solutions. As shown, considering average results, in one shipment we yielded stowage plans with more 700 units which can represent a saving of logistic costs. Thus, these new plans can be useful to ports replacing the ones currently used.

Simpler and faster heuristics exist, but the port authorities have the time to wait for a more complex approach to deliver a solution closer to optimality. They have pre-defined stored stowage plans of the known fleet and when a known ship is coming, they have the exact stowage plan for its holds. Besides, they also have a schedule of all shipments in the current year. So, when a different ship will arrive, they perform the stowage plans of the holds before it arrives at the port. Therefore, in one year, the time to define these new plans varies from months to days.

Now comparing WSP and pallet loading problems, our LagClus heuristic have solved instances with 273 units that are larger than the largest instances of the MPLP.

Points for further research are connected with the estimation of the ideal number of clusters given a time limit and a quality of the solution desired, and also the dynamic construction and management of conflict graphs to allow the treatment of instances of greater dimension.

We believe that LagClus can be useful to solve large-scale related practical problems like harvesting, anti-covering, frequency assignment and map labeling. Besides, it can be used in branch-and-price algorithms, or even in a column generation approach. 


\section{Acknowledgments}

The authors acknowledge the useful comments and suggestions of two anonymous referees.

This research was partially supported by Conselho Nacional de Desenvolvimento Científico e Tecnológico (CNPq). 


\section{REFERENCES}

Alvarez-Valdez R, Parreño F and Tamarit J M (2005 $)$. A tabu search algorithm for pallet loading problem. OR Spectrum 27(1): 43-61.

Alvarez-Valdez R, Parreño F and Tamarit J M (2005 $)$. A branch-and-cut algorithm for the pallet loading problem. Computer and Operations Research 32(11): 3007-3029.

Barnes F W (1979). Packing the maximum number of $m \times n$ tiles in a large $p \times q$ rectangle. Discrete Mathematics 26: 93-100.

Beasley J (1985). An exact two-dimensional non guillotine cutting tree search procedure. Operations Research 33: 49-64.

Bhattacharya R, Roy R and Bhattacharya S (1998). An exact depth-first algorithm for the pallet loading problem. European Journal of Operational Research 110: 610-625.

Birgin E G, Morabito R and Nishihara F H (2005). A note on an L-approach for solving the manufacturer's pallet loading problem. Journal of the Operational Research Society. To Appear.

Branch A E (1996). Elements of Shipping. Nelson Thornes: Cheltenham.

Christofides N and Whitlock C (1977). An algorithm for two-dimensional cutting problems. Operations Research 25: 30-44.

Dowsland K (1987). An exact algorithm for the pallet loading problem. European Journal of Operational Research 31: 78-84.

Dyckhoff H (1990). A typology of cutting and packing problems. European Journal of Operational Research 44: 145-159. 
Handabaka A R (1994). Gestão logística da distribuição física internacional (Choice of a chain of international physical distribution - A managerial approach). Maltese: São Paulo.

Held M and Karp R M (1971). The traveling salesman problem and minimum spanning trees: part II. Mathematical Programming 1: 6-25.

Herbert A and Dowsland K (1996). A family of genetic algorithm for the pallet loading problem. In: Osman I H and Kelly J P (eds). Metaheuristics: theory and applications. Kluwer Academic Publishers: Dordrecht, pp. 378-406.

Hodgson T (1982). A combined approach to the pallet loading problem. IIE Transactions 14(3): 176-182.

ILOG (2001) CPLEX 7.5 Reference Manual. 7.5v. OCopyright by ILOG: France.

Karypis G and Kumar V (1998). Multilevel k-way partitioning scheme for irregular graphs. Journal of Parallel and Distributed Computing 48(1): 96-129.

Lins L, Lins S and Morabito R (2003). An L-approach for packing ( $l, w)$-rectangles into rectangular and $L$-shaped pieces. Journal of the Operational Research Society 54: 777-789.

Morabito R. and Farago R. (2002). A tight lagrangean relaxation bound for the manufacturer's pallet loading problem. Studia Informatica Universalis 2(1): 57-76.

Morabito R, Morales S R and Widmer J A (2000). Loading optimization of palletized products on trucks. Transportation Research Part E 36: 285-296.

Morabito R and Morales S R (1998). A simple and effective heuristic to solve the manufacturer's pallet loading problem. Journal of the Operational Research Society 49: 819828. 
Narciso M G and Lorena L A N (1999). Lagrangean/Surrogate relaxation for generalized assignment problems. European Journal of Operational Research 114: 165-177.

Parker R G and Rardin R L (1998). Discrete Optimization. Academic Press: New York.

Pureza V and Morabito R (2006). Some experiments with a simple tabu search algorithm for the manufacturer's pallet loading problem. Computers and Operations Research 33(3): 804819.

Ribeiro G M and Lorena L A N (2005 $)$. Lagrangean relaxation with clusters and column generation for the manufacturer's pallet loading problem. Computers and Operations Research. To Appear.

Ribeiro G M and Lorena L A N (2005 $)$. Lagrangean relaxation with clusters for point-feature cartographic label placement problems. ECCO XVII post conference special issues. Submitted.

Scheithauer G and Terno J (1996). The G4-heuristic for the pallet loading problem. Journal of the Operational Research Society 47: 511-522.

Smith A. and De Cani P. (1980). An algorithm to optimize the layout of boxes in pallets. Journal of the Operational Research Society 31: 573-578.

Young-Gun G and Maing-Kyu K (2001). A fast algorithm for two-dimensional pallet loading problems of large size. European Journal of the Operational Research 134: 193-202. 


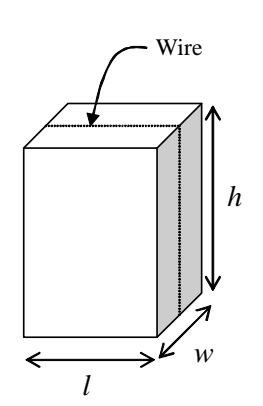

(a)

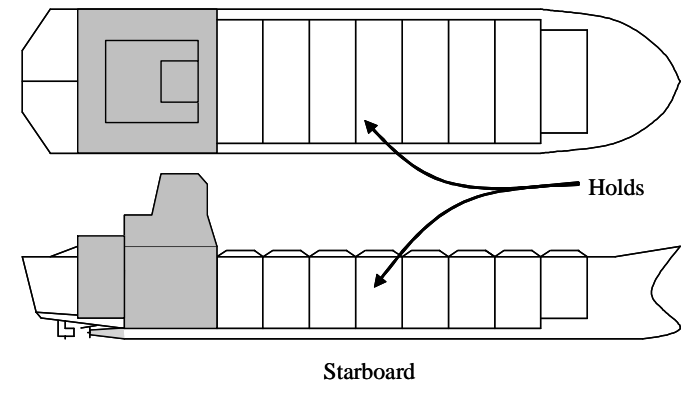

(b)

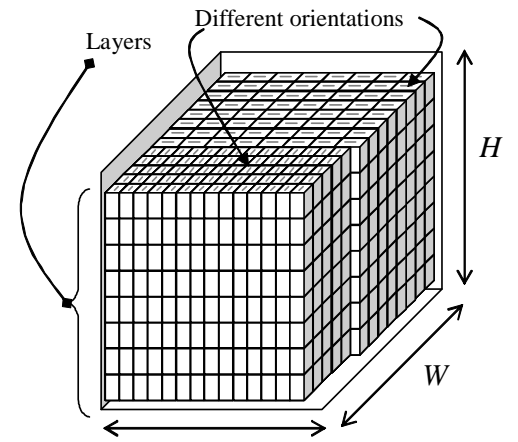

(c)

Figure 1: Woodpulp unit (a), layout of the holds (b), and (c) a stowage solution.

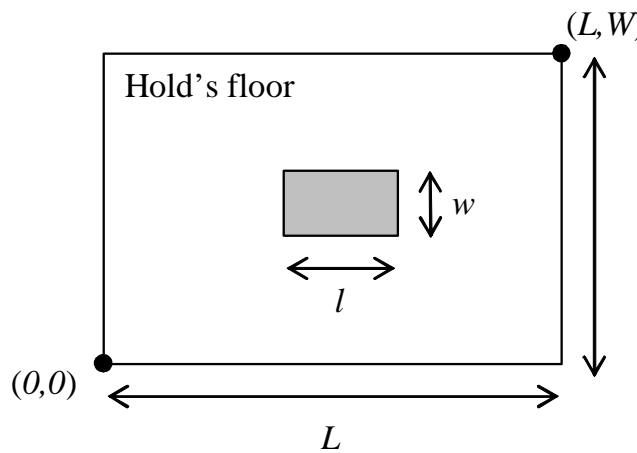

(a)

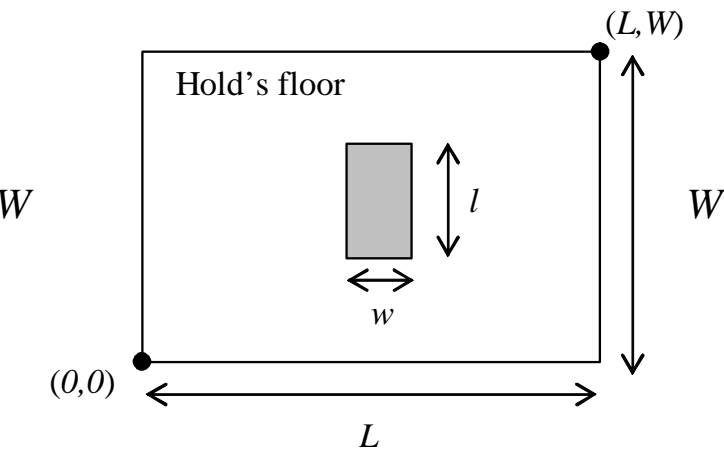

(b)

Figure 2: Possible orientations of one woodpulp unit. 


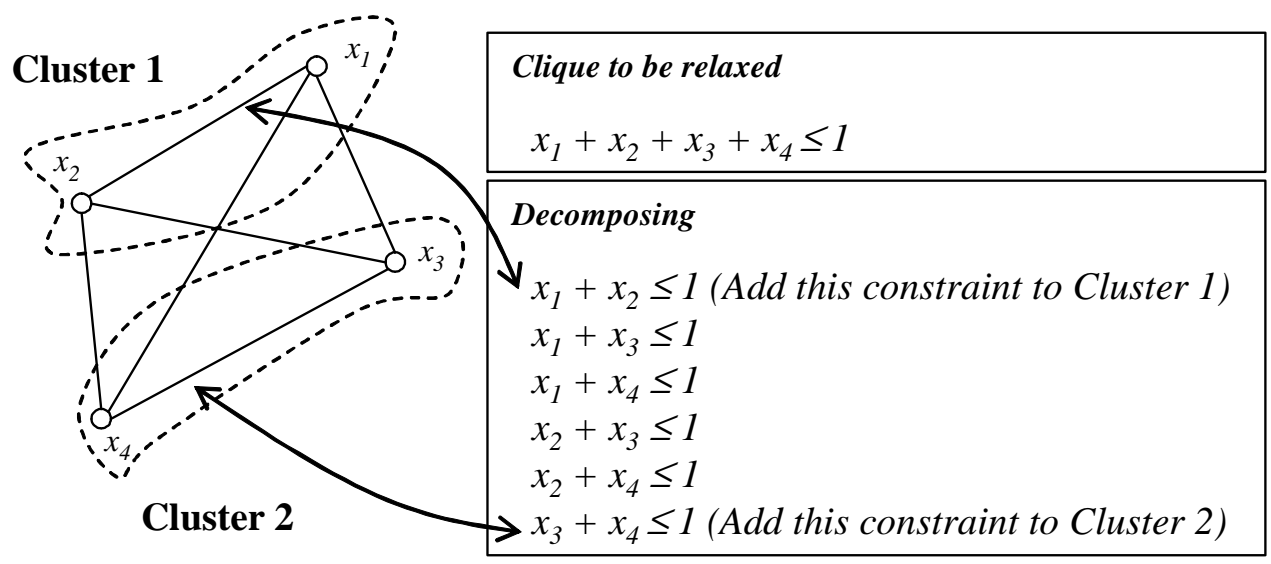

Figure 3: Example of how decompose a clique to be relaxed.
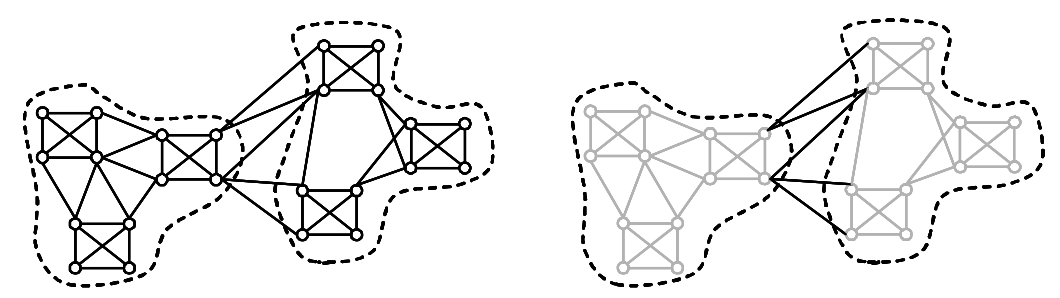

(a)

(b)
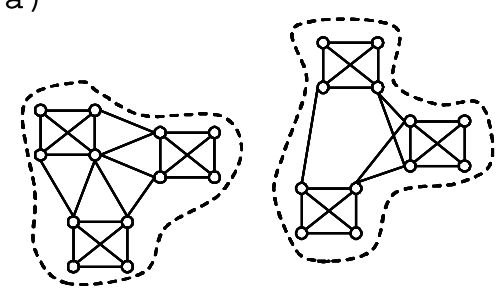

(c)

Figure 4: Lagrangean relaxation with clusters. (a) Conflict graph, (b) edges connecting the clusters, and (c) clusters or sub-problems.

Adapted from Ribeiro and Lorena (2005a) 
Verify and Improvement Heuristic - VI

1. Make feasible solution vector equal to the relaxed solution given by LagClus;

2. While not obtain a feasible solution Do 3. For each vertex $i$ in feasible solution vector, define the number of vertices $j$ that are in conflict with i;

4. Sort in decreasing order the feasible solution vector according to the number of conflicts; 5. Remove the first vertex from the feasible solution vector;

6. End while;

7. Verify among the other vertices not present in feasible solution and not present in that set removed from the feasible solution in step 2, if there are vertices that can be inserted in the feasible solution.

Figure 5 - Verify and improvement heuristic used in LagClus process.

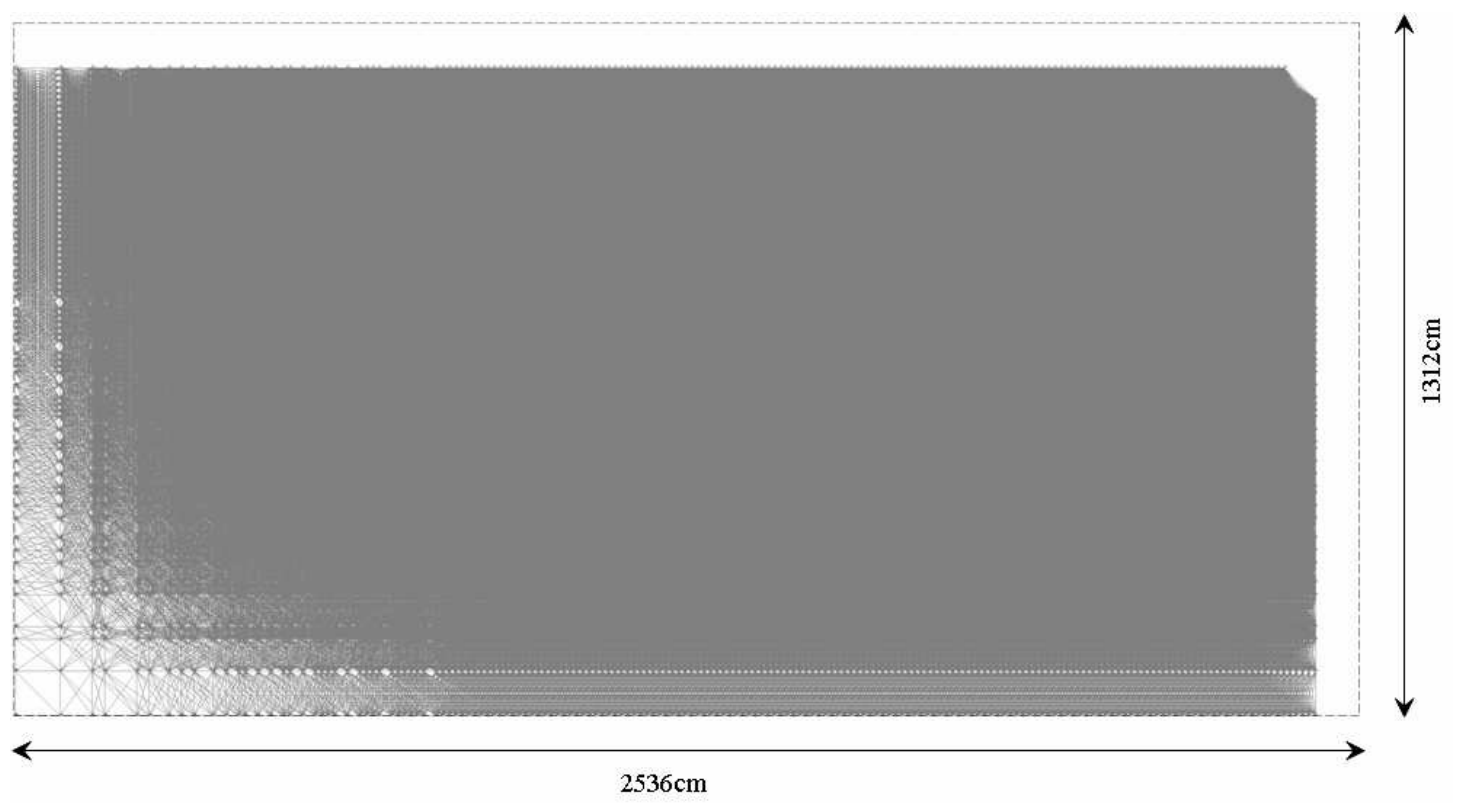

Figure 6 - Conflict graph generated for the instance L2: 22,870 vertices and 4,792,819 edges. 

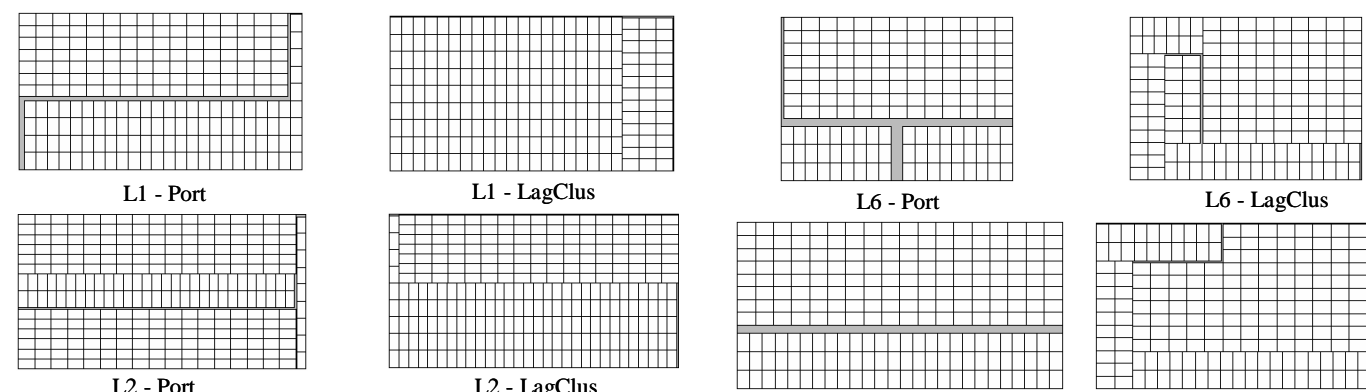

L6 - Port

L6 - LagClus
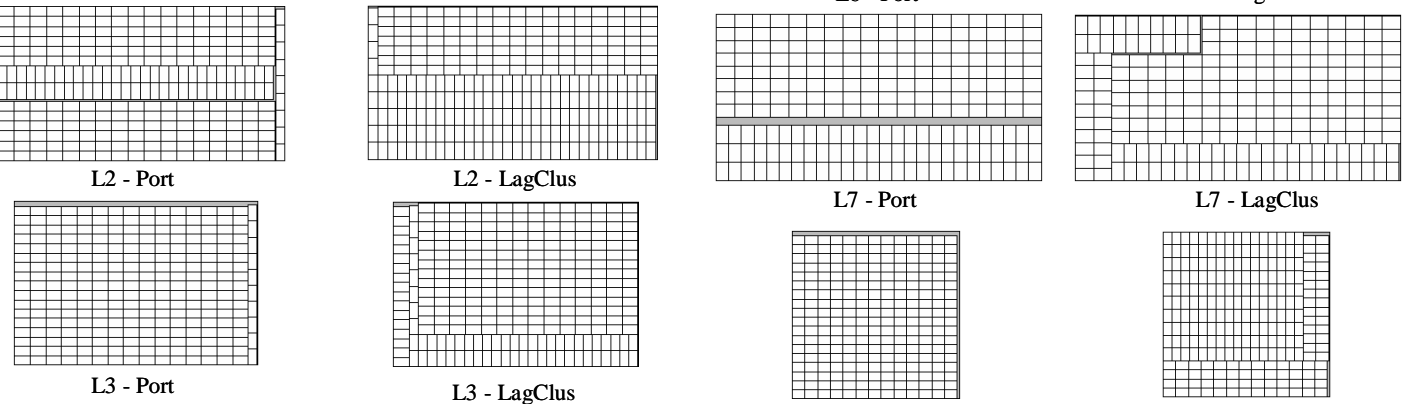

L3 - LagClus
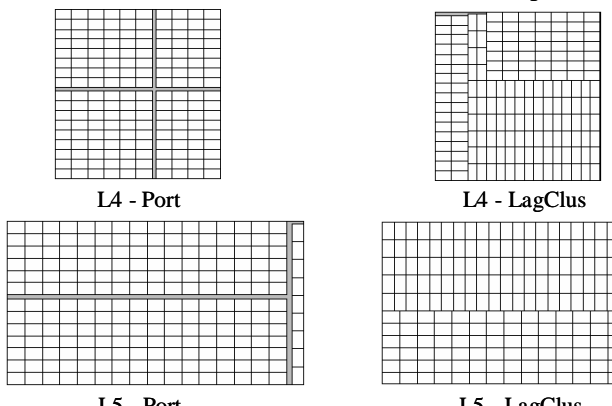

L4 - LagClus
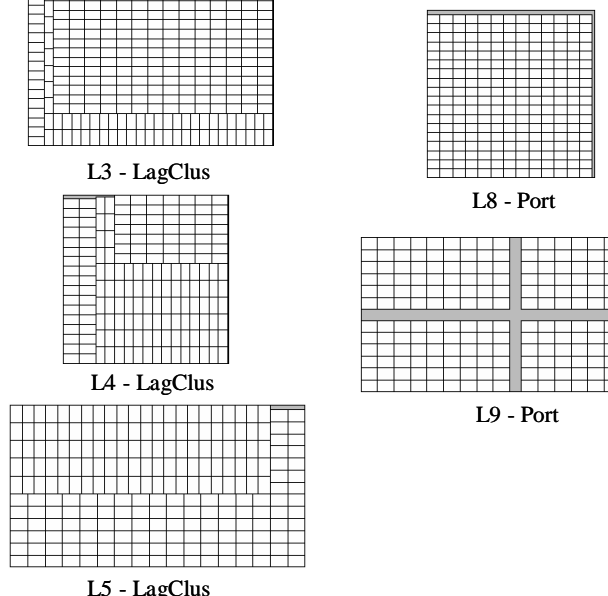

L8 - Port

L8 - LagClus
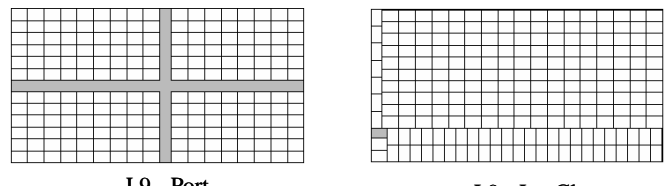

L9 - LagClus

L5 - Port

L5 - LagClus

Figure 7 - Solutions provided by LagClus for real instances of the WSP. 
Table 1: Real instances of the WSP obtained at Brazilian ports.

\begin{tabular}{|cccccccc|}
\hline \hline Instance & $\boldsymbol{L}(\boldsymbol{c m})$ & $\boldsymbol{W}(\boldsymbol{c m})$ & $\boldsymbol{H}(\boldsymbol{c m})$ & $\boldsymbol{L}(\boldsymbol{c m})$ & $\boldsymbol{w}(\boldsymbol{c m})$ & $\boldsymbol{h}(\boldsymbol{c m})$ & $\begin{array}{c}\text { Port's } \\
\text { Solution }\end{array}$ \\
\hline \hline L1 & 2296 & 1230 & 1600 & 136 & 94 & 184 & 213 \\
L2 & 2536 & 1312 & 1600 & 144 & 84 & 190 & 269 \\
L3 & 2252 & 1470 & 1652 & 144 & 84 & 190 & 265 \\
L4 & 1470 & 1458 & 1652 & 144 & 84 & 190 & 170 \\
L5 & 2296 & 1230 & 1600 & 135 & 92 & 183 & 217 \\
L6 & 1804 & 1230 & 1600 & 137 & 95 & 190 & 158 \\
L7 & 2466 & 1230 & 1600 & 137 & 95 & 190 & 222 \\
L8 & 1804 & 1750 & 1600 & 137 & 95 & 190 & 234 \\
L9 & 2426 & 1230 & 1640 & 137 & 95 & 190 & 204 \\
\hline \hline
\end{tabular}

The columns contain:

- Instance - Name of the instance;

- $\quad L, W$ and $H$ - Hold length, width and height, respectively;
- $l, w$ and $h-$ Woodpulp unit length, width and height, respectively;

- Port's Solution - Practical solution adopted by ports. 
Table 2: LagClus computational results for real instances of the WSP.

\begin{tabular}{|cccccccr|}
\hline \hline Instance & $\begin{array}{c}\text { Number of } \\
\text { Clusters }\end{array}$ & $\begin{array}{c}\text { Area } \\
\text { Bound }\end{array}$ & $\begin{array}{c}\text { Barnes } \\
\text { Bound }\end{array}$ & $\begin{array}{c}\text { Lower } \\
\text { Bound }\end{array}$ & $\begin{array}{c}\text { Upper } \\
\text { Bound }\end{array}$ & $\begin{array}{c}\text { GAP } \\
(\%)\end{array}$ & $\begin{array}{c}\text { Time } \\
(\mathbf{s})\end{array}$ \\
\hline \hline L1 & 30 & 220 & 220 & 219 & 220.01 & 0.46 & 4768 \\
L2 & 20 & 275 & 274 & 273 & 274.48 & 0.54 & 24637 \\
L3 & 30 & 273 & 273 & 271 & 272.52 & 0.56 & 9634 \\
L4 & 20 & 177 & 177 & 175 & 176.28 & 0.73 & 2889 \\
L5 & 30 & 227 & 227 & 226 & 228.66 & 1.16 & 6268 \\
L6 & 40 & 170 & 170 & 168 & 170.45 & 1.44 & 907 \\
L7 & 30 & 233 & 233 & 231 & 232.97 & 0.85 & 7460 \\
L8 & 30 & 242 & 242 & 240 & 242.77 & 1.14 & 16249 \\
L9 & 30 & 229 & 229 & 227 & 229.31 & 1.00 & 7384 \\
\hline \hline
\end{tabular}

The columns contain:

- Instance - Name of the instance;

- Number of clusters - Number of clusters used by LagClus;

- Area Bound - Area bound given by $\lfloor(L * W) /(l * w)\rfloor$ (where $\lfloor z\rfloor$ denotes rounding down to the nearest integer);

- Barnes bound - Bound provided by Barnes (1979);

- Lower bound - Lower bound found by VI heuristic;
- Upper bound - Upper bound provided by LagClus;

- GAP (\%) - Gap found between the lower and upper bound:

$$
\text { Gap }=\frac{(\text { Upper Bound }- \text { Lower Bound })}{\text { Upper Bound }} * 100 ;
$$

- Time - Time in seconds elapsed by LagClas reaching some of the stop conditions; 
Table 3: Results for the real instances of the Brazilian ports.

\begin{tabular}{|c|c|c|c|c|c|c|}
\hline \multirow[b]{2}{*}{ Instance } & \multicolumn{3}{|c|}{ Number of woodpulp units by tier } & \multicolumn{3}{|c|}{ Number of woodpulp units by hold } \\
\hline & $\begin{array}{c}\text { Port's } \\
\text { Solution }\end{array}$ & $\begin{array}{l}\text { Lower } \\
\text { Bound }\end{array}$ & Difference & $\begin{array}{c}\text { Number of } \\
\text { tiers }\end{array}$ & $\begin{array}{c}\text { Port's } \\
\text { Solution }\end{array}$ & $\begin{array}{l}\text { Lower } \\
\text { Bound }\end{array}$ \\
\hline$\overline{\mathrm{L} 1}$ & 213 & 219 & 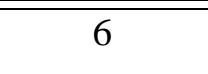 & $\overline{88}$ & 1704 & 1752 \\
\hline L2 & 269 & 273 & 4 & 8 & 2152 & 2184 \\
\hline L3 & 265 & 271 & 6 & 8 & 2120 & 2168 \\
\hline $\mathrm{L} 4$ & 170 & 175 & 5 & 8 & 1360 & 1400 \\
\hline L5 & 217 & 226 & 9 & 8 & 1736 & 1808 \\
\hline L6 & 158 & 168 & 10 & 8 & 1264 & 1344 \\
\hline L7 & 222 & 231 & 9 & 8 & 1776 & 1848 \\
\hline L8 & 234 & 240 & 6 & 8 & 1872 & 1920 \\
\hline L9 & 204 & 227 & 23 & 8 & 1632 & 1816 \\
\hline Average & 216.89 & 225.56 & 8.67 & 8 & 1735.11 & 1804.44 \\
\hline
\end{tabular}

The columns contain:

- Instance - Name of the instance;

- Port's Solution - Practical solution adopted by ports;

- Lower bound - Lower bound found by VI heuristic;

- Difference - The difference calculated as: Lower Bound - Port's Solution;

- Number of tiers - Number of tiers is calculated as: $\lfloor H / h\rfloor$. 


\section{Caption for Figures and Tables}

Figure 1: Woodpulp unit (a), layout of the holds (b), and (c) a stowage solution.

Figure 2: Possible orientations of one woodpulp unit.

Figure 3: Example of how decompose a clique to be relaxed.

Figure 4: Lagrangean relaxation with clusters. (a) Conflict graph, (b) edges connecting the clusters, and (c) clusters or sub-problems. Adapted of Ribeiro and Lorena (2005a).

Figure 5 - Verify and improvement heuristic used in LagClus process.

Figure 6 - Conflict graph generated for the instance L2: 22,870 vertices and 4,792,819 edges.

Figure 7 - Solutions provided by LagClus for real instances of the WSP.

Table 1: Real instances of the WSP obtained at Brazilian ports.

Table 2: LagClus computational results for real instances of the WSP.

Table 3: Results for the real instances of the Brazilian ports. 